\title{
可调控高表达钙调素细胞模型的建立 与初步分析*
}

\author{
任 宁 卢智俊 柳惠图 \\ （北京师范大学生物系, 北京 100875）
}

\section{关链词 钻调素、表达载体 pCAM、细胞转化}

钔调素 (Calmodulin 简称 $\mathrm{CaM}$ ) 是广泛存在于各类真核细胞中、具有调节细胞生长、分 化和癌变作用的蛋白 ${ }^{[1]}$. Means 等发现钙调素含量的增高是细胞进入 $s$ 期的限速因子 ${ }^{[2]}$. 肿 瘦细胞中钙调素含量高于相应的正常细胞已被多次证实 ${ }^{[3]}$. 但因研究方法的局限, 缺少有效 的钙调素刺激剂以提高细胞内 $\mathrm{CaM}$ 的水平,观察过量䥻调素对细胞的作用效应.针对这一问 题, 我们构建了生理剂量 $(1 \mu \mathrm{mol} / \mathrm{L})$ 的糖皮质激素地塞米松 (Dexamethasone, DXM) 调 控的、钙调素高表达的细胞模型, 并对此细胞模型在钻调素高表达状态下生长特性及与转化相 关性进行初步检验和比较, 以探讨钙调素的表达与细胞转化的直接相关性.

\section{一、材料和方法}

1. 细菌、细胞及其培养 大肠杆菌 RRI、HB101、JM109 的培养条件基本按 Maniatis 等在文献 [4]中叙述的方法.所有细胞培养基均为 RPMI-1640, $10 \%$ 小牛血清(Gibco 产品).

2. 质粒 Blue PGEM3 为美国 Promega 公司产品, $\mathrm{pSV} 2$ neo 为 H. Land 博士 (USA) 送, 钙调素 $\mathrm{cDNA} p \mathrm{pCB} 12 、 \mathrm{pCB} 15^{[9]}$ 为美国 Baylor 医学院黎志豪教授赠送, PMTVLTR 为中国科学院动物研究所沈孝宙教授赠送.

3. 内切酶、佟饰酶及各种化学试椡购自美国 Sigma、BRL 等公司, $\mathrm{Na}^{125}$ 购自中国 原子能科学研究院, 钙调素系作者从猪脑中提取纯化并经免疫取得抗血清.

4. 基因转移及细盷株的䈂选与建立 基因转移采用磷酸钻沉淀法, 目标基因与标记基 因 pSV2neo 共转染基本上按 Person 等叙述的方法 ${ }^{161}$. 用 G418 篮选生长的克隆, 分别消化, 扩大培养进行鉴定.

5. 蛋白质聚丙烯酰胺电泳及 Western blotting SDS-聚丙烯酰胺电泳采用 Laemmli 系统 ${ }^{[7]}$. Westeris blotting 基本上按改进的 Towbin 等方法 ${ }^{[3]}$.

6. CaM 的放免分析 (Radioimmunoassay, RIA) 取 $10^{4}$ 细胞加人含有碘标钙调 素 $(100 \mathrm{pg})$ 的样品管中, 和一抗温育后加人 Igsorbin, 离心后测定其放射性. 同时做出标准曲 线,根据标准曲线计算出 $\mathrm{CaM}$ 含量.

7. 软琼脂上成集落性实验加热 $2 \times 1640$ 培养基与 $0.5 \% \mathrm{Agar}$, 待温度下降后迅速

本文 1989 年 10 月 25 日收到. 1990 年 3 月 15 日收到修改稿.

- 国家自然科学基金资助项目. 
将 $10^{4}$ 细胞与两者混匀, 铺于塑料平皿上, 待凝固后置于 $37^{\circ} \mathrm{C}$ 培养, 两周后观察结果.

\section{二、结果与讨论}

1. 真核衰达戟体 pCAM 的制备与箁选 因为钻调素 $\mathrm{cDNA} p \mathrm{pB} 12$ 与表达载体 PMTVLTR 的连接为平头末端连接, 连接频率很低, 为了构建真核表达载体 pCAM, 我们选 择了中间载体—Blue pGEM3, 将 pCB12 与 pGEM3 载体重组, 首先构建了质粒 pSC. 用 $\mathrm{Taq} 1$ 将 pSC 彻底酶切并平头化后, 回收 $0.8 \mathrm{~kb}$ 片段; 同时将 $\mathrm{pMMTV} \mathrm{dhfr}$ 经酶切后所得 的大片段与来自 $\mathrm{pSC}$ 的 $0.8 \mathrm{~kb}$ 片段连接, 转化感受菌 RRI, 经筛选与鉴定后将所构建的表达 $\mathrm{CaM}$ 的真核表达载体命名为 pCAM. pSC 和 pCAM 的具体构建程序和内切酶鉴定结果分 别见图 1A、B.
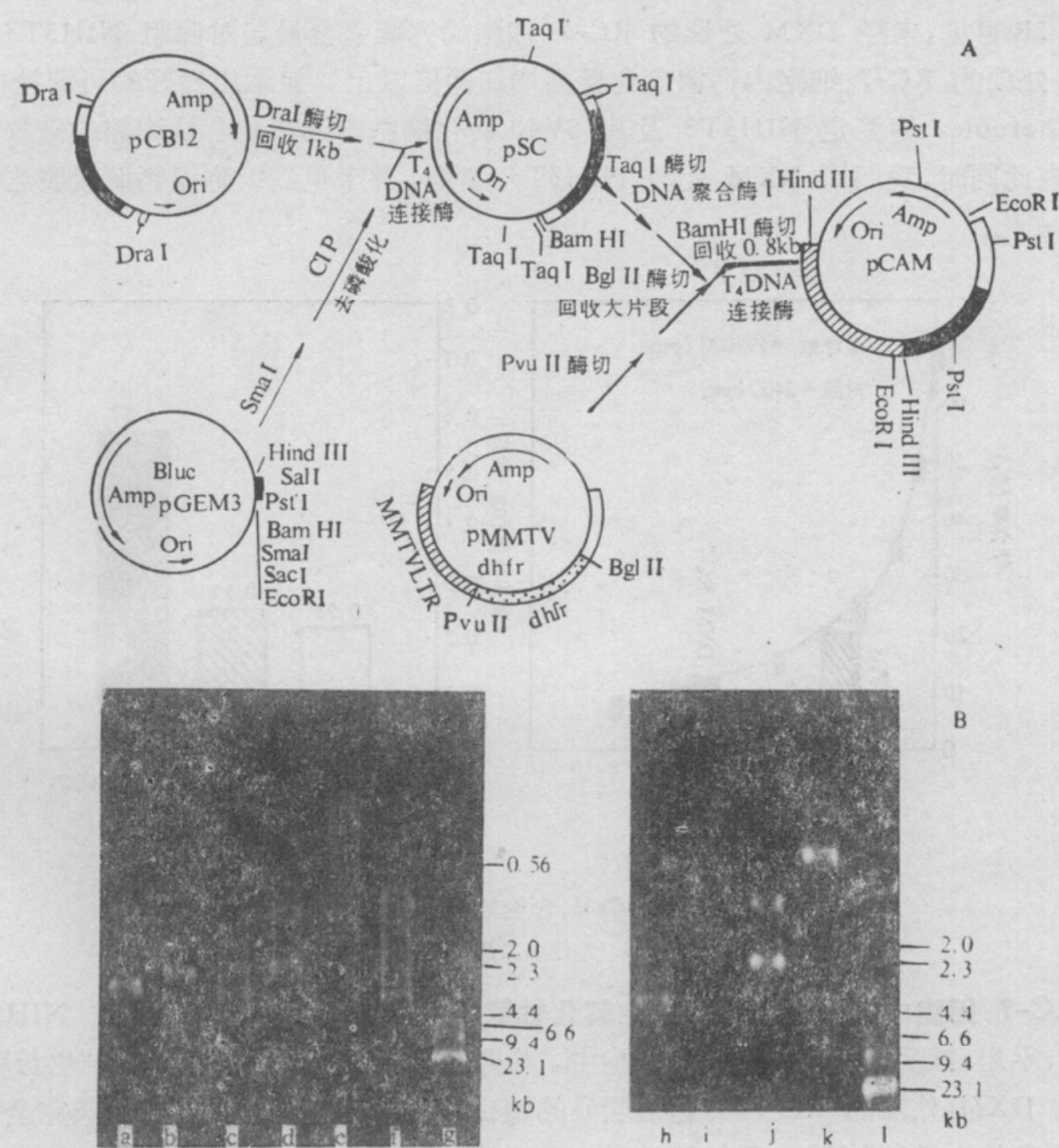

图 1

A. 重组质柆 $\mathrm{pSC}$ 和 $\mathrm{pCAM}$ 的构建程序; B. 各种内切酶对重组质柆 $\mathrm{pSC}$ (左)和 pCAM (右)的签定.

a. pCB12-Dral; b. pGEM3-BamHI; c. pSC-BamHI: d. pSC-Sac I-BamHI;

e. pSC-EcoR1; f. pSC-HindllI; g. 1. 分子量标准 ( $\lambda$-DNA-HindIII):

h. pCAM-Hind III; i. pCAM-EcoRI: i. pCAM-Pst I; k. pSC-Taql 
2. 可调控钙调素衰达细胞模型的建立 用磷酸钲沉淀法 将 pCAM 与 pSV2neo (10:1) 共转染 NIH 3T3 细胞, 经 G418 節选, 共得到约 200 个阳性克隆, 我们选择了 $\mathrm{i} 2$ 个克隆进一 步篮选, 分别命名为 RC-1-RC-12.

\section{RC 系列细胞克隆的进一步䇻选与钙调素可控性的鉴定 以 NIH $3 \mathrm{~T} 3$ 细 胞为对} 照, 我们比较了.上述部分 $\mathrm{RC}$ 系列细胞克隆在生理剂量 $1 \mu \mathrm{mol} / \mathrm{L}$ 地塞米松 (DXM) 诱导 $24 \mathrm{~h}$ 后钙调素表达水平, 通过 Western blotting 实验, 可见在条带 $17 \mathrm{KDa}$ 处,来源于 RC-7 等细 胞克隆的蛋白质显色深度均高于其对照组 NIH3T3 细胞(图未显示). 表明 RC-7 等 细 胞 克隆在诱导剂作用下具有较高的钻调素表达水平. 显示了载体 $\mathrm{pCAM}$ 能在 NIH3T3 细胞 中表达出结构、大小正常的鸡钻调素. 为了进一步测定细胞内钻调素的精确含量, 我们用 1 $\mu \mathrm{mol} / \mathrm{L}$ DXM 处理 RC-7 细胞 24h 后, 将细胞裂解, 进行放免分析实验, 并与对照组细胞进 行比较, 图 $2 \mathrm{~A}$ 为放免分析结果, 用这一结果绘制标准曲线, 并计算出处理细胞内的钻调素含 量. 从图 2B 可见,未经 DXM 处理的 RC-7 细胞的䥻调素含量与对照组 NIH3T3 近似,但 经 DXM 处理的 RC-7 细胞内钻调素含量则增加两倍以上, 显示出良好的可调控性. 这个 结果与 Chafoulea 等测定 NIH3T3 及其 SV40 转化细胞内䥻调素含量增加的倍数关系是一 致的 ${ }^{[3]}$. 在此同时, 我们用 DXM 处理 NIH3T3 细胞, 并未见到细胞内钻调素表达水平具有 可探测的变化.
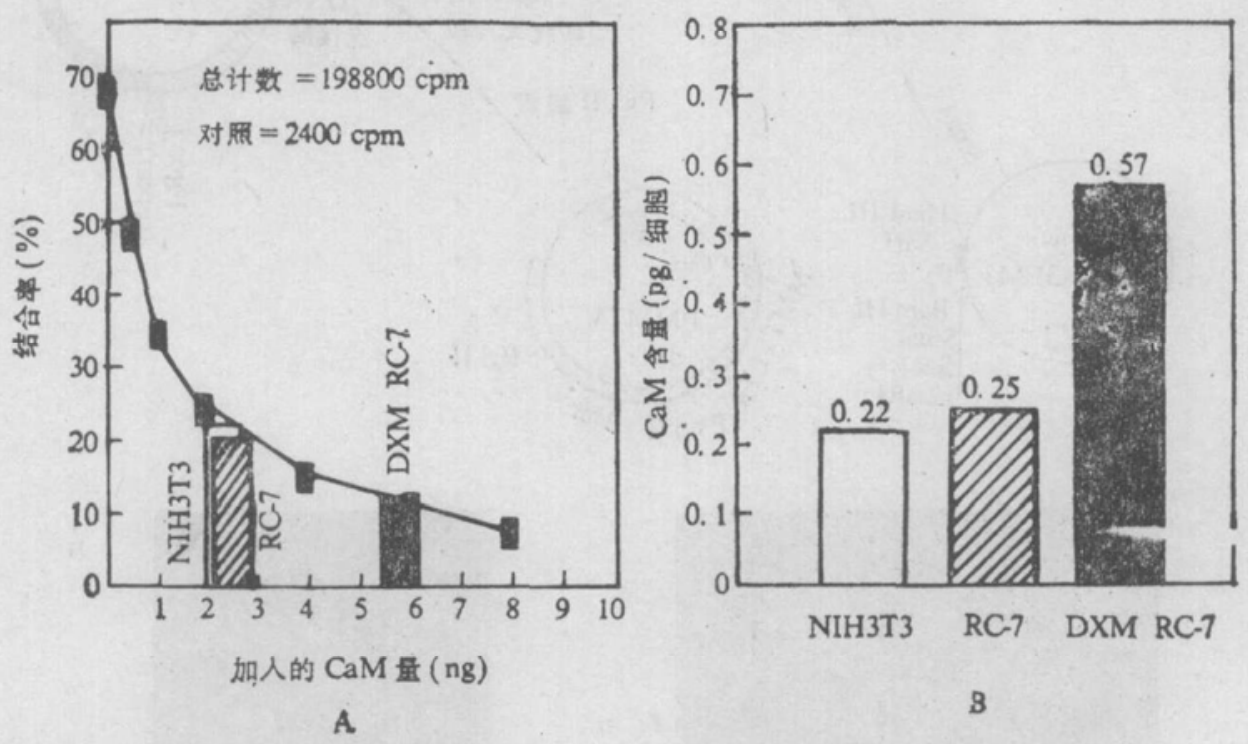

图 2 测定细胞内 $\mathrm{CaM}$ 含量的竞争性放射免度分析

A. 测量曲线；B. 为最小二乘法算出的浓度方块图

\section{RC-7 细胞中钔调素表达与细胞转化性能相关性之初步分析 正常 NIH3T3 细胞} 生长铺展、平坦, 核质比小; 在 RC-7 细胞中, 可见生长密度较高, 但未见成堆生长现象(图 3 A); 但用 DXM 处理的 RC-7 细胞呈明显的转化表型, 细胞核质比增大, 生长完全丧失密度 依赖性, 呈岛状生长(图 3B). 我们还对另一株正常大鼠肾细胞 NRK 进行了转染, 发现也有 类似的形态变化. 看来钙调素的过表达导致培养细胞出现转化特征是带有普遍性的.

为了进一步分析经 DXM 处理的 RC-7 细胞确实具有转化细胞的性能, 我们用含 $1 \mu \mathrm{mol} /$ L DXM 的 $0.2 \%$ 涼脂培养 RC-7 细胞, 三周后长出直径约 $0.2-0.5 \mu \mathrm{m}$ 的克隆 (图 4). 但 NIH3T3 细胞和未经 DXM 处理的 RC-7 细胞在软琼脂上培养并未见克隆. 进一步观察到 


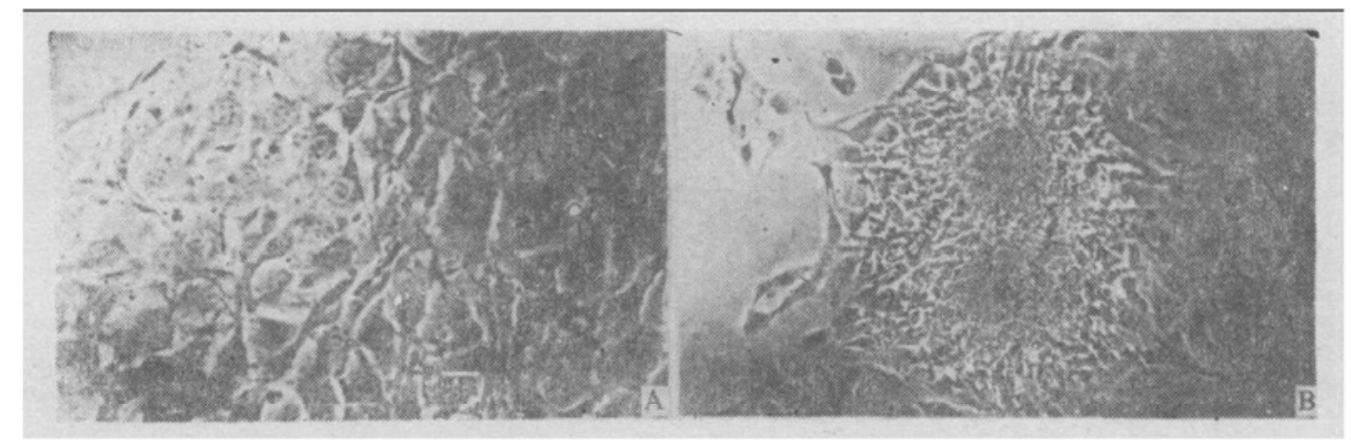

图 3

A. 未加 DXM 诱导的 RC-7 细跑形态与 NIH3T3 细胞相似、平坦、舒展,未呈堆积状生长;

B. 加人 $1 \mu \mathrm{mol} / \mathrm{L}$ DXM 诱导 48h 后的 RC-7 细胞其形态类似癌基因 V-sis 转化的 NIH3T3 细胞 (结果末显示), 细盷堆积成多层, 呈身状生长 $(\times 100)$

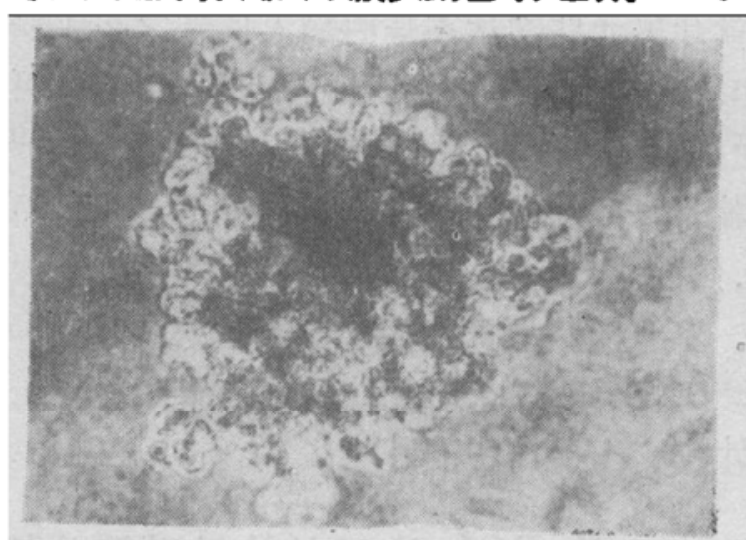

图 $41 \mu \mathrm{mol} / \mathrm{L}$ DXM 诱导 RC-7 细胞在 $0.25 \%$ 软琼脂半固体培养基上长出高比例集落 集落直径为 $0.2-0.5 \mathrm{~mm}, \mathrm{NIH} 3 \mathrm{~T} 3$ 细胞和未加 DXM 诱导的 RC-7 细胞未能长出集落 (培养时间 21 天, $\times 100$ )

这些高表达细胞形成集落的速率与比率和 sis 癌基因转化细胞相似(实验数据未显示).

同时,我们也观察了细胞微丝的变化, 初步看到经 DXM 处理的 RC-7 细胞微丝荧光弥 散,表明微丝处于解聚状态(图未显示).

通过上述初步实验与分析,表明我们成功地构建了具有良好、可调的高表达钻调素的细胞 模型. 并且发现在诱导剂作用下钙调素高表达时, 就能使这些非转化细胞获得了部分转化表 型. 本工作不仅为研究钙调素在细胞内的调节作用提供了有效的细胞模型, 更为重要的是首 次报道了钙调素水平的提高可能直接作用于细胞转化表型, 为研究细胞转化与癌化机理提供 了一个新的探讨线索.

\section{考文献}

[1] Veigl, M.L. et al., Biochem. Biophys. Acta, 738 (1984), 21-48.

[2] Chafoulea. J. G. et al., Cell, 36 (1984), 73-81.

[3] Chafoulea, J. G. et al., Proc. Natl. Acad. Sci., 78 (1981), 996-1000.

[4] Maniatis, T. et al., Moiecular Cloning, Cold Spring Harbor Laboratory, New York, 1982. $68-85$.

[5] Putkey, J. A. et sl., J. Biol. Chem.. 258 (1983), 11864-11870.

[6] Persons, D, A. et al., Cell, 52 (1988), 447-458.

[7] Laemmli, U. K., Nature, 227 (1970), 680-685.

[8] Towbin, H. et al., Proc. Natl. Acad. Sci.,76 (1979), 4350-4354. 